\title{
Physique des mouvements rapides chez les plantes
}

Yoël Forterre ${ }^{(1)}$ (yoel.forterre@univ-amu.fr), Philippe Marmottant ${ }^{(2)}$,

Catherine Quilliet ${ }^{(2)}$ et Xavier Noblin ${ }^{(3)}$

(1) Laboratoire IUSTI, CNRS UMR 7343, Aix Marseille Université, 13453 Marseille

(2) Laboratoire Interdisciplinaire de Physique, CNRS UMR 5580, Université de Grenoble-Alpes

(3) Laboratoire de Physique de la Matière Condensée, CNRS UMR 7336, Université Nice Sophia Antipolis

\section{Dépourvues de muscles, \\ certaines plantes mettent en œuvre des mouvements dont la fulgurance est comparable à celle des animaux.}

Nous montrons dans cet article que beaucoup de ces mouvements, nécessités par la reproduction ou la nutrition, ont la même base physique : une instabilité mécanique qui libère de l'énergie élastique stockée.

Deux grands types d'instabilités mécaniques sont utilisés par les plantes pour amplifier la vitesse de leur mouvement : les ruptures solides ou liquides (cavitation) pour la propulsion des graines ou des spores de fougères, et les instabilités de flambage élastique pour les pièges des plantes carnivores, telles que la Dionée ou l'utriculaire.
Les plantes bougent, et pas seulement sous l'effet du vent ou de la croissance. Certaines ont mis au point au cours de leur évolution des mécanismes de mouvements propres étonnamment rapides, avec des vitesses de pointe $(\sim 10 \mathrm{~m} / \mathrm{s})$ et surtout des accélérations (milliers de $g$ ) supérieures à ce qu'on peut rencontrer dans le monde animal. Ces mouvements rapides, de nature quasi explosive, sont généralement associés à des fonctions importantes comme la reproduction (propulsion de pollen chez certaines orchidées, mouvements explosifs de dispersion de graines), la défense contre des prédateurs (repliement du Mimosa Pudica) ou la nutrition (pièges des plantes carnivores). Ils fascinent les scientifiques depuis les observations de Linné et Darwin, et continuent à générer des questions sur la vitesse maximale de processus physiologiques importants comme le transport d'eau et d'ions à travers les membranes cellulaires, la modification des propriétés mécaniques de la paroi cellulaire ou les signaux associés à la mécano-perception (Forterre, 2013).

Dans cet article, nous exposons la physique des ingénieux mécanismes utilisés par les plantes pour se mouvoir, sans l'aide d'aucun muscle.

\section{À l'origine des mouvements : osmose et évaporation}

Pour comprendre comment une plante peut produire des mouvements, il est nécessaire de faire une brève plongée dans sa structure intime. Une différence fondamentale entre les cellules animales et végétales est que ces dernières sont entourées d'une paroi rigide, constituée principalement de cellulose.
Cette paroi permet aux cellules végétales de soutenir une grande pression d'eau interne, appelée pression de turgescence. Une pression de turgescence de l'ordre de 4 à 8 bars est habituelle dans les cellules de plantes bien hydratées, et peut même atteindre jusqu'à 40 bars (40 fois la pression atmosphérique !) dans certaines cellules spécialisées. La rigidité des cellules végétales leur permet à l'inverse, dans certaines situations, de soutenir des pressions internes d'eau négatives (ce qui signifie que le fluide, au lieu de pousser, tire les parois vers l'intérieur par le biais de ses forces cohésives, voir encadré 1). L'exemple le plus connu est la sève des arbres, qui circule sous tension dans les vaisseaux conducteurs avec une pression négative pouvant atteindre -100 bars.

Physiquement, l'origine de ces valeurs extrêmes de pressions provient de phénomènes d'osmose ou d'évaporation (voir encadré 2). Ces flux d'eau entre la plante et l'extérieur gonflent ou dégonflent les cellules de la plante. Il en résulte une pression de turgescence, qui entraîne un mouvement mécanique à l'échelle macroscopique. Certains de ces mouvements correspondent à une déformation irréversible de la paroi des cellules, comme les mouvements de croissance par rapport à la gravité (fig. 1a). D'autres sont associés à une déformation réversible, comme le repliement des feuilles de la plante sensitive Mimosa Pudica induit par osmose (fig. 1c), ou l'ouverture et la fermeture des écailles de pommes de pin sous l'effet de changement d'humidité de l'air (fig. 1b). 


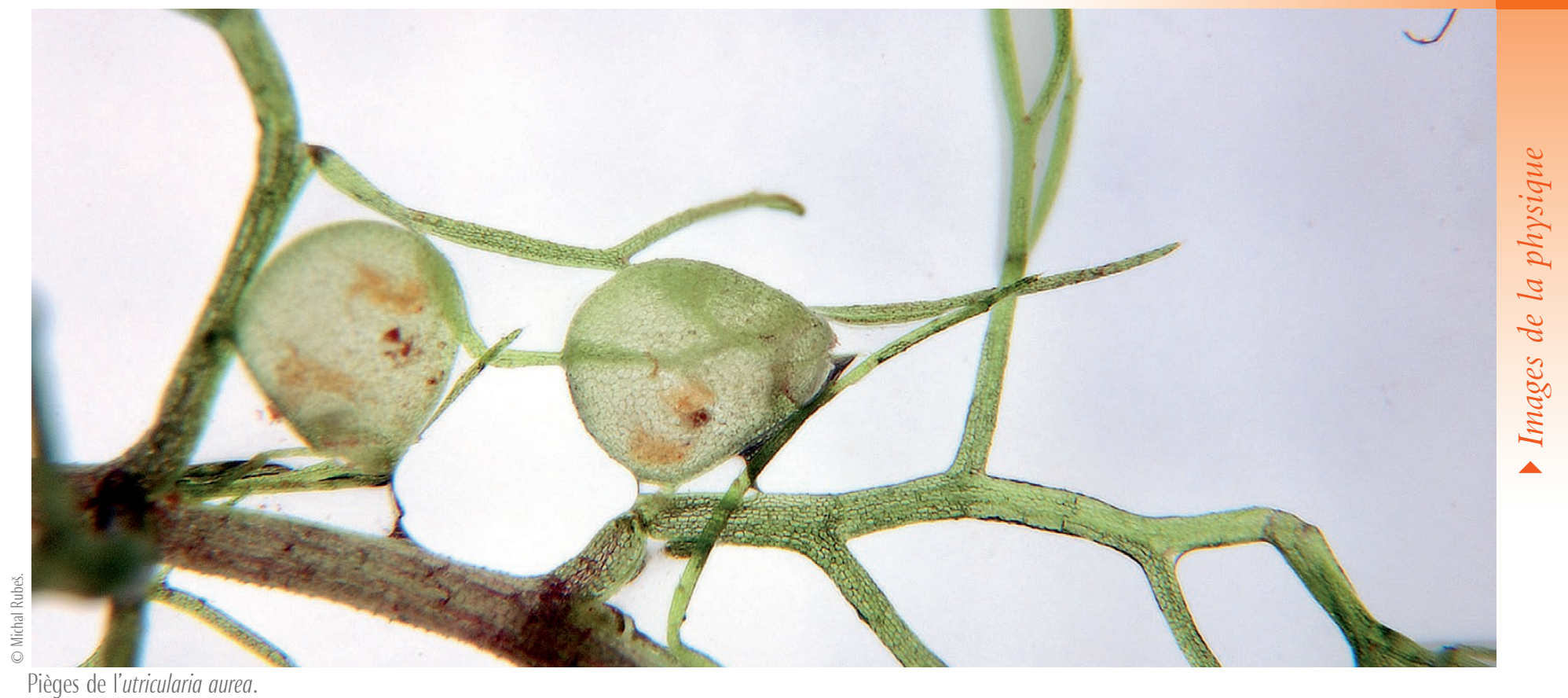

\section{Pression négative et cavitation}

Autant la pression d'un gaz est forcément positive, autant celle d'un liquide peut être négative, à condition que l'on s'écarte de l'état d'équilibre thermodynamique. Considérons l'équation d'état d'une mole de fluide décrit par l'équation de Van der Waals :

$P=[R T /(V-b)]-\left(a / V^{2}\right)$, où $P$ est la pression, $V$ le volume, $R$ la constante des gaz parfaits et $T$ la température. Les paramètres a et $b$ sont reliés aux interactions entre molécules et représentent un écart aux gaz parfaits. En traçant les isothermes (fig. E1a), on trouve que la pression peut devenir nettement négative dans une certaine gamme de volumes. La construction de Maxwell permet de déterminer les paliers entre liquide et vapeur : la pression, toujours positive, y vaut $P_{\text {vap }}(T)$ et correspond à l'état d'équilibre (pointillés sur la figure). En partant du liquide, on peut cependant diminuer la pression à des valeurs inférieures à $P_{\text {vap }}$ et même à 0 , tant qu'aucune bulle de vapeur n'apparaît. Les molécules du liquide s'éloignent légèrement les unes des autres et, tels des ressorts, elles résistent en tirant les parois du récipient au lieu de les pousser (fig. E1b). C'est la cohésion des molécules entre elles et avec les parois qui permet d'exercer cette pression négative, représentée par le terme $\left(a / V^{2}\right)$. Les pressions négatives observées dans de l'eau peuvent atteindre plusieurs centaines de fois la pression atmosphérique.

Ces états persistent tant qu'aucun germe ou défaut ne vient provoquer la nucléation d'une bulle. Cela nécessite de fournir de l'énergie pour dépasser une barrière liée à la tension de surface entre la vapeur et le liquide. La compétition entre énergie de surface et énergie de volume implique que seules les bulles avec des rayons supérieurs au $\mathrm{nm}$ peuvent croître. Une fois nucléée, une telle bulle de cavitation croît violemment, avec un temps caractéristique de l'ordre de la microseconde, jusqu'à ce que l'on retrouve l'équilibre thermodynamique entre liquide et vapeur (fig. E1c).

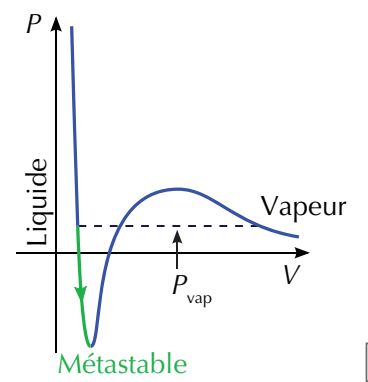

Liquide sous tension

Liquide et vapeur en équilibre
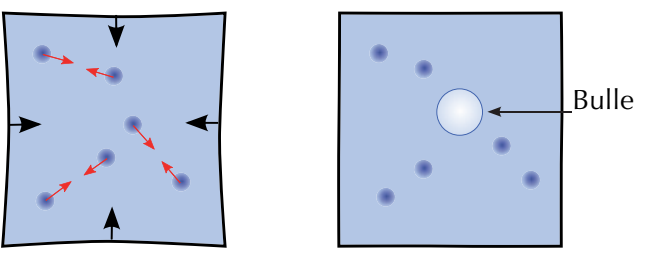

b
Osmose, évaporation et pression de turgescence chez les plantes

Les phénomènes d'osmose et d'évaporation sont cruciaux pour bien comprendre l'origine des grandes gammes de pression rencontrées dans les cellules végétales. Comme toute cellule, la cellule végétale est entourée d'une membrane perméable à l'eau mais imperméable à certains solutés présents dans la cellule (sels, sucres). D'un point de vue entropique, ces solutés cherchent à occuper le plus d'espace possible; ils ont donc tendance à " pomper » l'eau pure qui se trouve à l'extérieur de la cellule, de l'autre côté de la membrane semi-perméable, jusqu'à ce que les potentiels chimiques de l'eau de part et d'autre de la membrane soient égaux. À l'équilibre, il existe alors dans la cellule une surpression $\Delta P=C R T$ égale à la pression osmotique, où $C$ est la concentration en soluté dans la cellule. Pour une concentration de $0,2 \mathrm{~mol}^{-\mathrm{I}^{-1}}$, on trouve que la surpression dans la cellule (la pression de turgescence) est de 5 bars, une valeur typique des cellules végétales.

Le phénomène opposé se produit lorsqu'une cellule est en contact avec l'air. Le potentiel chimique de l'eau dans l'air est en général beaucoup plus bas que dans la cellule, ce qui signifie que l'eau s'évapore de la cellule. L'équilibre n'est atteint que s'il existe une dépression dans la cellule égale à :

$\Delta P=\left(R T / V_{w}\right) \ln [h]<0$, où $V_{w}$ est le volume molaire partiel de l'eau liquide, et $h$ le taux d'humidité relative dans l'air. Pour une humidité relative de $90 \%$, cette dépression d'équilibre vaut -140 bars ! L'eau est alors sous une forte tension et dans un état métastable, ce qui peut donner lieu à de la cavitation (voir encadré 1).

E1. (a) Isotherme dans le diagramme P-V d’un fluide réel (bleu) ; vert : zone métastable ; pointillés : équilibre liquide-vapeur. (b) Un liquide peut soutenir des pressions négatives (tension), grâce aux forces d'interaction attractives entre molécules (flèches rouges). (c) L'apparition d’une bulle relâche ces tensions. 


\section{Limite physique des mouvements purement hydrauliques : le temps poroélastique}

Les mouvements des plantes, malgré leur diversité, ont donc tous le même point de départ : un transport d'eau piloté par osmose ou par évaporation. Ce mécanisme commun impose une contrainte physique forte : la vitesse d'un mouvement basé uniquement sur un échange d'eau est nécessairement limitée par le temps de transport de l'eau à travers le tissu. On peut montrer que le transport de l'eau à travers un tissu poreux et élastique comme le tissu végétal est un processus diffusif, qui opère sur un temps $\tau_{p}=L^{2} / D$, où $L$ est la distance de transport et $D$ un coefficient de diffusion effectif qui tient compte des propriétés élastiques et hydrauliques du milieu.
Le temps $\tau_{p}$, appelé temps poroélastique, fixe donc la vitesse maximale des mouvements basés uniquement sur le transport d'eau chez les plantes. Pour illustrer cette idée, nous avons tracé sur la figure 1 la durée $\tau$ de plusieurs mouvements du règne végétal en fonction de la taille typique de leur tissu en mouvement. On constate que la durée des mouvements associés à la croissance (fig. 1a) ou de ceux liés à un changement d'humidité de l'air ambiant (fig. 1b), se trouvent au-dessus de la frontière $\tau=\tau_{p}$, ce qui est compatible avec leur nature purement " hydraulique». C'est également le cas du repliement des feuilles du Mimosa Pudica (fig. 1c). Bien que ce mouvement soit beaucoup plus rapide que les précédents (de l'ordre de la seconde), il reste compatible avec un transport d'eau car il opère sur une distance beaucoup plus petite.
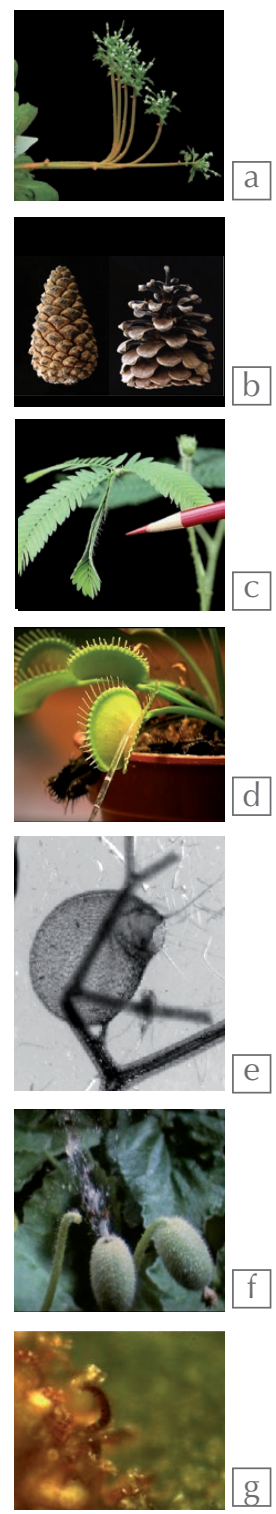

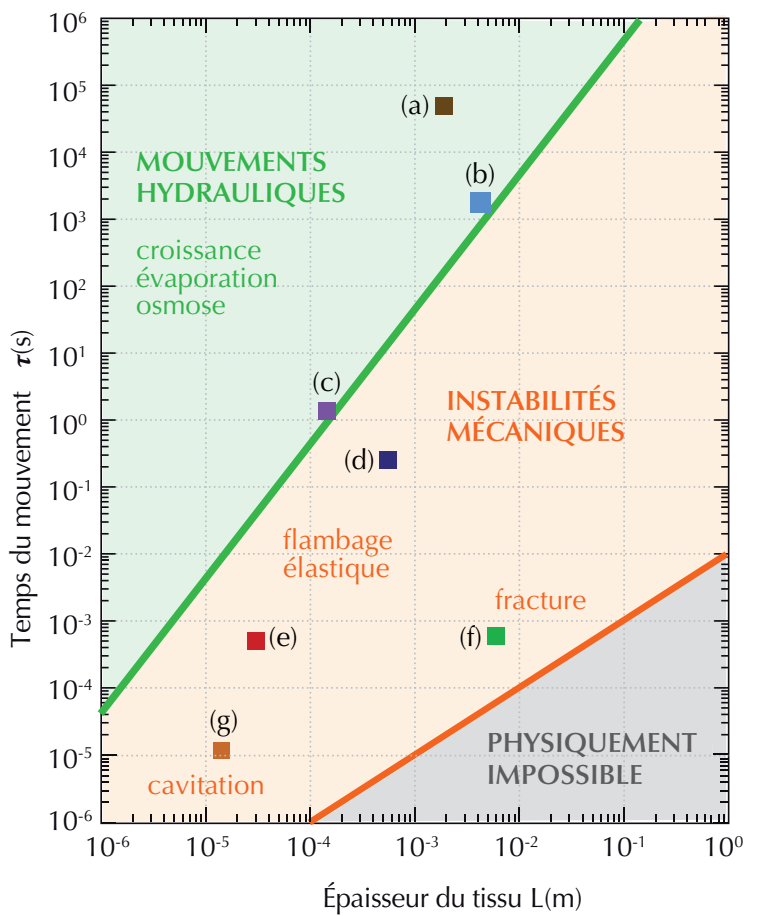

1. Classification physique des mouvements chez les plantes en fonction de leur durée $\tau$ et de la taille typique $L$ sur laquelle opère le mouvement (d'après Skotheim et Mahadevan, 2005). La frontière $\tau=\tau_{p}$ (trait vert) donne le temps poroélastique (le mouvement hydraulique le plus rapide possible) en prenant un coefficient de diffusion moyen pour l'eau dans les tissus végétaux $D \sim 10^{-8} \mathrm{~m}^{2} \mathrm{~s}^{-1}$. La frontière $\tau=\tau_{j}$ (trait orange) donne le temps inertiel (le mouvement élastique le plus rapide possible) en prenant une densité $\rho=1000 \mathrm{~kg} \mathrm{~m}^{-3}$ et un module de Young $\mathrm{E}=10 \mathrm{MPa}$.

(a) Mouvement de croissance en réponse à la gravité.

(b) Mouvement hygroscopique des écailles de la pomme de pin.

(c) Repliement des feuilles du Mimosa Pudica par osmose.

(d) Plante carnivore Dionée.

(e) Plante carnivore utriculaire.

(f) Propulsion de graines chez le concombre d'âne (Ecballium elaterium).

(g) Mouvement de catapulte des spores de fougère.

\section{Comment augmenter la vitesse des mouvements : instabilités mécaniques}

Si les végétaux s'appuyaient uniquement sur le transport d'eau pour bouger, ils ne pourraient pas produire des mouvements sur une échelle de temps plus petite que celle donnée par le temps poroélastique. Pourtant, la figure 1 montre que de nombreuses plantes franchissent largement cette limite hydrodynamique et rivalisent avec les plus rapides mouvements rencontrés chez le vivant. Comment font ces champions du règne végétal pour atteindre de telles vitesses? Nous allons voir que leur stratégie commune est d'utiliser une instabilité mécanique, c'est-à-dire la libération rapide d'énergie élastique au-delà d'un certain seuil. Deux grands types d'instabilités mécaniques sont utilisés par les plantes pour produire des mouvements rapides : les instabilités de flambage élastique pour les pièges des plantes carnivores telles que la Dionée ou l'utriculaire, et les ruptures solides ou liquides (cavitation) pour la propulsion des graines ou des spores de fougères. Dans la suite de l'article, nous discutons ces deux grandes classes de mécanismes.

\section{Instabilité de flambage élastique : les plantes carnivores Dionée et utriculaire}

La plante carnivore Dionée (Dionaea muscipula) - qualifiée de "merveille de la nature " par Darwin - présente l'un des mouvements rapides les plus connus du règne végétal. Son piège est constitué de deux lobes attachés à leur base pour former une sorte de mâchoire (fig. 2a). La face interne de chaque lobe contient des poils mécano-sensibles. Lorsque ces poils sont touchés par l'insecte, un signal électrique est généré. Le piège se ferme alors en quelques dixièmes de secondes, ce qui est trop rapide pour être expliqué uniquement par un transport d'eau (fig. 1).

L'étude de la géométrie et de la dynamique de fermeture du piège a permis de montrer que la Dionée utilise une instabilité élastique pour amplifier sa vitesse de fermeture. Les lobes du piège ont en effet une géométrie de coque mince, bombés vers l'extérieur dans l'état ouvert et bombés vers l'intérieur dans l'état fermé (fig. 2b,c). Lorsque le piège est déclenché, les lobes essaient de se courber vers l'intérieur en changeant 
activement leur courbure naturelle par un mécanisme osmotique ou de croissance rapide encore mal compris. Cependant, en raison de la forme de coque des lobes, ce changement de courbure nécessite le franchissement d'une barrière d'énergie élastique car la déformation d'une coque mince est très coûteuse d'un point de vue énergétique (voir encadré 3). De l'énergie élastique s'accumule donc dans le système jusqu'à ce que la barrière élastique soit franchie. Les lobes "flambent " alors brutalement vers l'intérieur, refermant le piège autour de sa proie.

Le même type d'instabilité a été récemment dévoilé chez une autre plante carnivore : l'utriculaire. Moins connue que la Dionée, l'utriculaire (du genre Utricularia) est pourtant présente dans les marais de la plupart des continents. Ses pièges à succion sont d'une sophistication remarquable. Chaque piège est une petite outre élastique, qui se met lentement en dépression sous l'action de glandes qui pompent l'eau vers l'extérieur (fig. 2d). Il faut environ une heure pour " armer" ainsi le piège. Le piège est muni d'une porte flexible, initialement fermée, sur laquelle sont fixés des poils sensitifs.

Le déclenchement de la succion est réalisé en un temps record d'une milliseconde, grâce à un mécanisme d'instabilité élastique similaire à celui de la Dionée. En effet, tout comme les lobes de la Dionée, la porte de l'utriculaire est une coque élastique. Initialement bombée vers l'extérieur, elle résiste à la différence de pression. Le toucher d'un poil sensitif diminue cette résistance, sans doute suite à la propagation d'un signal électrique : le flambage élastique de la porte se déclenche, ce qui inverse complètement la forme de la porte et l'ouvre vers l'intérieur (fig. 2e,f). L'eau environnante est rapidement aspirée, avec une accélération de $600 \mathrm{~g}$, enrichie de petits animaux qui ne peuvent nager contre ce courant (fig. 2d).

\section{Instabilité de cavitation : la propulsion des spores de fougères}

Si des mouvements rapides peuvent servir à la nutrition (Dionée et utriculaire), beaucoup sont utiles à la reproduction et assurent la propulsion et l'éjection de graines (voir par exemple le Concombre d'âne, fig. 1f), de pollen ou de spores. Parmi ceux-là, l'un des plus élégants est sans doute le catapultage des spores de fougères (fig. 3a). Chez la plupart des fou-
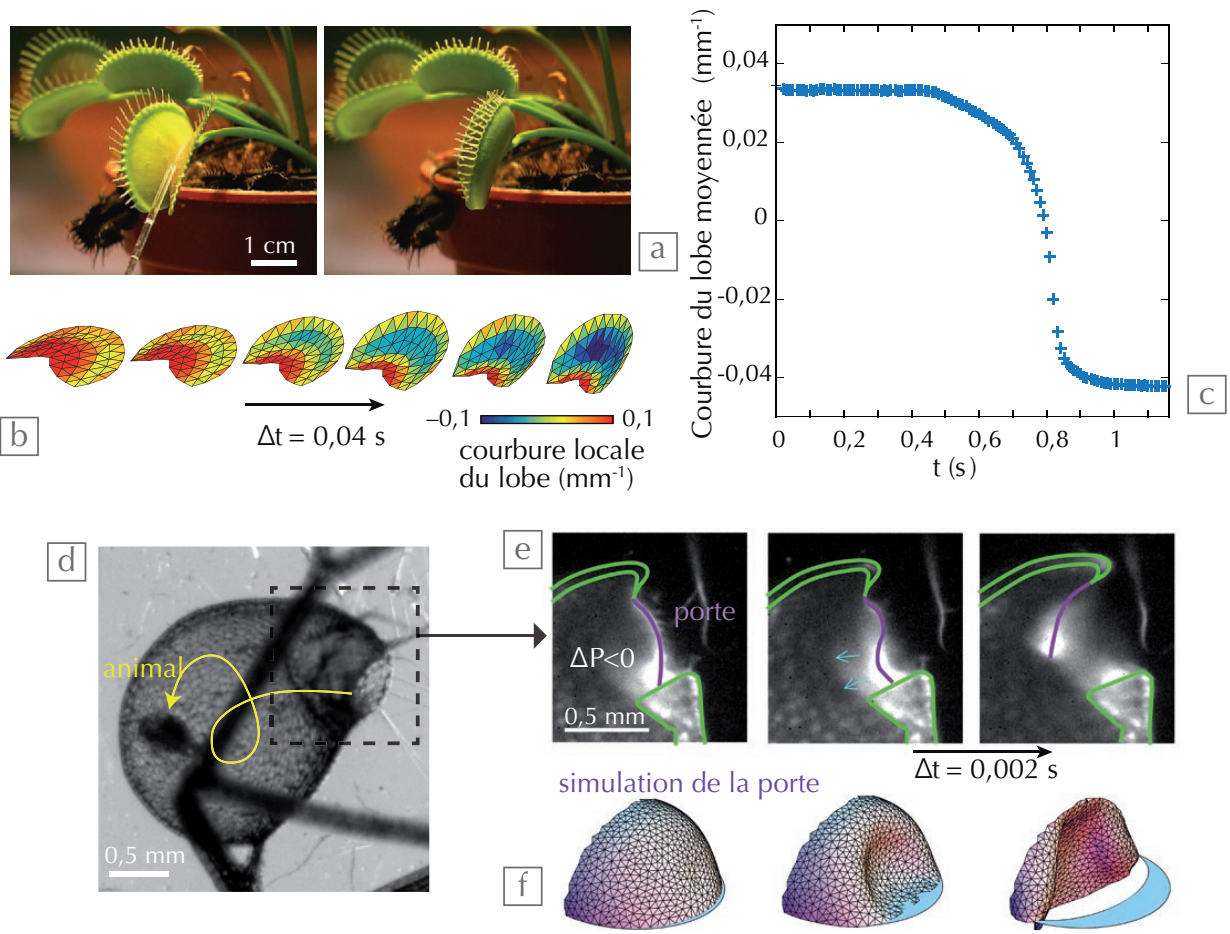

2. Instabilité de flambage élastique chez les plantes carnivores Dionée (a-c) et utriculaire (d-f).

(a) Piège de la Dionée dans l'état ouvert (gauche) et fermé (droite).

(b) Forme 3D d'un lobe du piège durant la fermeture, mesurée à l'aide d'un dispositif stéréoscopique

$(\Delta t$ : intervalle de temps entre deux images successives).

(c) Courbure moyenne du lobe en fonction du temps ; le piège a été déclenché à $t=0$.

(d) Piège de l'utriculaire et trajectoire d'un petit crustacé d'eau douce aspiré juste après l'ouverture de la porte.

(e) Imagerie en nappe laser de la porte du piège lors de l'ouverture.

(f) Simulation numérique du flambage de la porte élastique.

(Figures a-c d'après Forterre et al., 2005. Figures d-f d'après Vincent et al., 2011.)

\section{Instabilité de flambage d'une coque mince élastique}

L'instabilité d'une coque mince élastique est l'archétype d'une instabilité mécanique provenant d'une contrainte purement géométrique. Cet objet possède deux positions d'énergie locale minimale : la coque courbée d'un côté ou de l'autre (fig. E2). Pour passer de l'une à l'autre, il est nécessaire de franchir une position intermédiaire dans laquelle la coque est presque plane. Or, on sait depuis les travaux du mathématicien F. Gauss qu'il est impossible de transformer une surface sphérique en une surface plane sans changer son aire. Ce changement d'aire est très coûteux du point de vue de l'énergie élastique et entraîne l'existence d'une barrière d'énergie. Ainsi, lorsque l'on essaie de retourner une coque mince, celle-ci préfère résister dans un premier temps, ce qui se traduit généralement par des modifications d'aire locales et peu perceptibles qui accumulent de l'énergie élastique, plutôt que de se courber dans l'autre sens.

Cependant, si l'on augmente la force appliquée, il arrive un moment où l'accumulation d'énergie permet de franchir la barrière d'énergie élastique. La coque « flambe » alors brutalement de l'état initial vers l'état final de courbure opposée, relâchant rapidement l'énergie élastique.
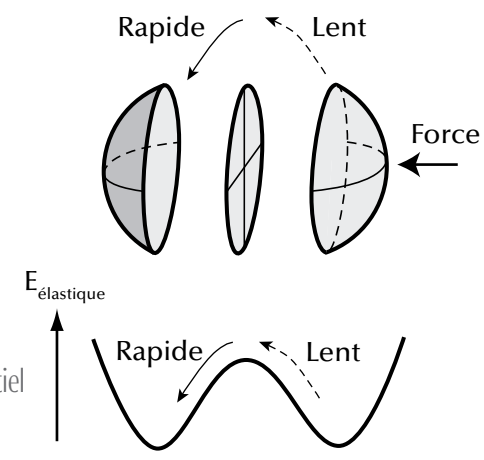

E2. Instabilité de flambage d'une coque mince et allure du potentiel élastique associé. 


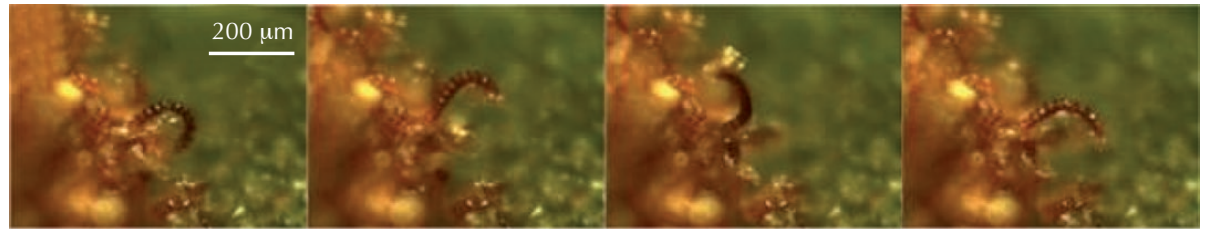

1

2

3

4.

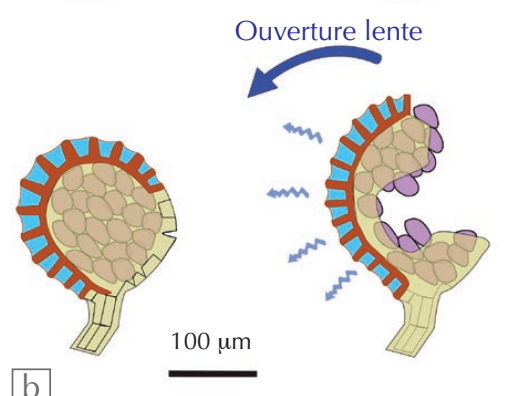

Catapulte inertielle

Relaxation poroélastique
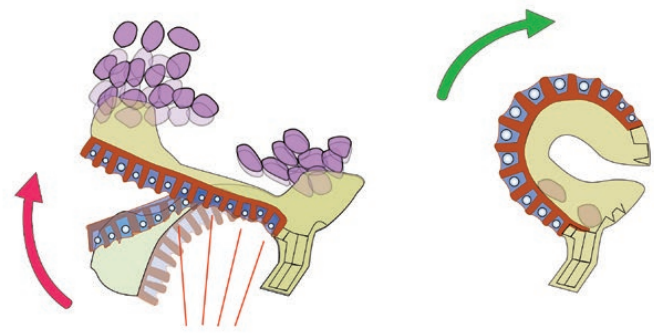

Bulles de cavitation

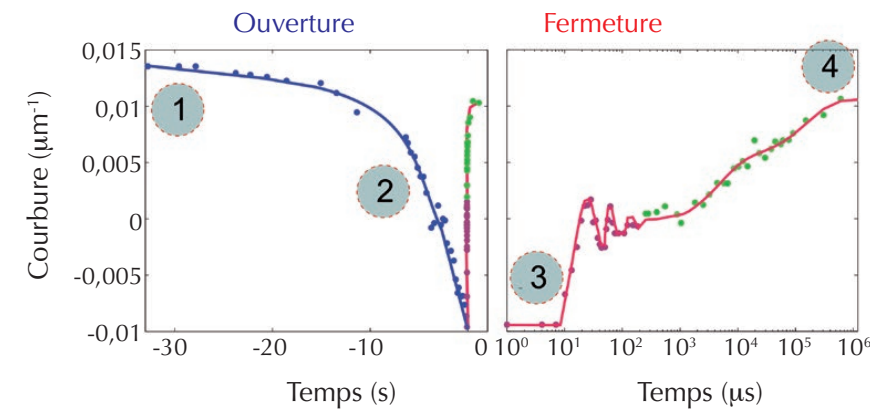

3. Catapulte par cavitation du sporange de fougère.

(a) Ouverture et fermeture d'un sporange ayant déjà envoyé ses spores.

(b) Mécanisme d'éjection en quatre étapes, correspondant aux quatre photos de la figure a (sur la troisième photo, on ne voit que la position avant le dédenchement).

(c) Courbure moyenne du sporange en fonction du temps pour l'ouverture lente (courbe bleue) et la fermeture rapide (courbe rouge). En échelle logarithmique, on perçoit mieux les oscillations inertielles puis la relaxation poroélastique (d’après Noblin et al., 2012).

\〉

gères, les spores sont réunies en amas sous les feuilles et se développent dans des capsules sphériques, les sporanges, possédant une «crête " en forme d'anneau constituée d'une douzaine de cellules remplies d'eau (fig. 3b). Lorsque le sporange est exposé à l'air, l'eau s'évapore des cellules de l'anneau et la pression dans les cellules devient négative (voir encadré 1). Cette pression négative tire sur les parois des cellules et entraîne l'ouverture de l'anneau car les parois sont asymétriques : fines du côté de l'interface avec l'air et épaisses du côté de l'interface avec le sporange (fig. 3b). Lorsque la tension dans l'eau devient trop grande (environ -90 bars), des bulles de cavitation apparaissent dans les cellules. Leur croissance brutale relaxe l'énergie élastique emmagasinée dans les parois et entraîne la fermeture rapide du sporange, et l'éjection des spores comme dans une catapulte.
Le mécanisme d'éjection est compris dans les grandes lignes depuis plus d'un siècle, mais de nombreuses questions restaient en suspens jusqu'à récemment. Un point en particulier intriguait. Pour être fonctionnelles, les catapultes médiévales et romaines utilisaient une butée, qui permettait de stopper à mi-course le bras de la catapulte et, ainsi, d'éjecter le projectile avant qu'il ne retourne au sol. En l'absence d'un tel dispositif, comment le sporange réussit-il à propulser efficacement les spores ? Des expériences récentes utilisant une caméra rapide ont permis d'élucider la question. La dynamique de fermeture du sporange est en fait non triviale et constituée de deux phases (fig. 3c). Dans la première phase, qui ne dure que quelques dizaines de microsecondes après la cavitation (il s'agit probablement du mouvement le plus rapide chez les végétaux), le mouvement du sporange est inertiel, résultant de la transformation d'énergie élastique en énergie cinétique. Cette phase est si rapide que l'eau piégée dans les parois poreuses de l'anneau n'a pas le temps de se redistribuer. La pression d'eau dans les pores augmente alors brutalement, ce qui bloque transitoirement l'anneau à mi-course et permet l'éjection des spores à plus de $10 \mathrm{~m} / \mathrm{s}$. Dans un deuxième temps, une fois les oscillations inertielles amorties, la pression d'eau relaxe lentement et le sporange se referme complètement en quelques centaines de millisecondes. Ainsi, ce système constitué de quelques cellules et d'eau est suffisant pour remplir toutes les fonctions d'une catapulte, de la phase d'armement liée à l'évaporation de l'eau au déclenchement par cavitation et enfin à la butée permettant l'éjection des projectiles, grâce au comportement poroélastique de la paroi végétale.

\section{Conclusion}

En conclusion, les plantes présentent des stratégies remarquables, liées à des instabilités mécaniques de flambage ou de cavitation, pour atteindre des vitesses beaucoup plus rapides que celles générées par leur moteur interne, qui est limité par les flux d'eau avec un temps de réponse poroélastique. Notons qu'il existe tout de même une limite ultime à ces stratégies, à savoir le temps inertiel de mise en mouvement des masses accélérées : cette limite se traduit par l'existence d'un temps inertielélastique $\tau_{i}=L / c$, où $L$ est la taille de l'objet et c la vitesse des ondes élastiques, en deçà duquel les mouvements sont physiquement impossibles (fig. 1).

Ces plantes ne sont pas que des curiosités de nostalgiques de la leçon de choses. À l'heure où le biomimétisme permet de forcer certaines barrières technologiques, les mouvements sans muscles constituent une source d'inspiration prometteuse pour la réalisation de systèmes artificiels en microfluidique et robotique.

\section{Références}

Y. Forterre et al., Nature 433 (2005) 421.

J. M. Skotheim et L. Mahadevan, Science 308 (2005) 1308.

0 . Vincent et al., Proceedings of the Royal Society B $\mathbf{2 7 8}$ (2011) 2909.

X. Noblin et al., Science 335 (2012) 1322.

Y. Forterre, Journal of Experimental Botany 64 (2013) 4745. 